\title{
Impact of the left anterior descending artery wrapping around the left ventricular apex on cardiac mechanics in patients with normal coronary angiography
}

\author{
Hala Mahfouz Badran ${ }^{1 *}$ (D), Waleed Abdou Ibrahim', Tamer Alaksher ${ }^{2}$ and Ghada Soltan ${ }^{1}$
}

\begin{abstract}
Background: We examined the impact of left anterior descending (LAD) wrapping on left ventricular (LV) mechanics in patients with normal coronary angiography. Seventy-one patients with evidence of normal coronary angiography (LAD wrapping: $n=52,73 \%)$ and LAD non-wrapping $(n=19,27 \%)$ were included in the study. Using 2D-strain imaging, we measured LV longitudinal and circumferential (circ) strain $\left(\varepsilon_{\text {sys }}\right)$, systolic strain rate $\left(S R_{\text {sys }}\right)$, early $\left(S R_{e}\right)$ and atrial (SRa) diastolic SR, LV electromechanical dyssynchrony (TTP-SD), and LV twist and torsion in study groups.

Results: No significant difference in age, gender, body surface area (BSA), or ejection fraction (EF\%) between groups. LAD-wrapping group showed higher deceleration time (DT) $(P<0.0001)$, global longitudinal $\varepsilon_{\text {sys }} \%(P<0.02)$, circ $\mathrm{SR}_{a}$ at the basal segments $(P<.02)$, circ $\mathrm{SR}_{\text {sys }}$ and $\mathrm{SR}_{e}$, and $\mathrm{SR}_{\mathrm{a}}(P<0.0001)$ at the apical segments and apical rotation compared with the non-wrapped group. LV twist was correlated negatively with LV electromechanical dyssynchrony $(r=.25, P<0.03)$ and positively with longitudinal $\varepsilon_{\text {sys }}(r=.47, P<.0001)$, circ $\varepsilon_{\mathrm{sys} \%}(r=.55, P<.0001)$, circ SR $\mathrm{S}_{\text {sys }}(r=.23$, $P<.05)$, and circ SRe $(r=.55, P<.0001)$. Using multivariate regression analysis, DT: OR 0.932, Cl 0.877-0.991, and $P<$ 0.02 and circ at atrial diastole $\left(\mathrm{SR}_{\mathrm{a}}\right): \mathrm{OR} 0.000, \mathrm{Cl} .000-.271$, and $P<0.03$ were independent predictors of $\mathrm{LAD}$ wrapping around LV apex.

Conclusion: Wrapped LAD is associated with better myocardial relaxation and rotational mechanics in patients with normal coronary angiography. This could explain the worse prognosis in such population when LAD occlusion acutely emerges.
\end{abstract}

Keywords: Left anterior descending wrapping, LV mechanics, Speckle tracking

\section{Background}

Nowadays, coronary artery disease represents $31 \%$ of deaths and the most important cause of morbidity and mortality all over the world [1]. The left anterior descending coronary artery (LAD) supplies blood flow to the greater part of the cardiac muscle. Nevertheless, the amount of myocardium supplied with LAD differs according to its length, and consequently, the site of coronary artery occlusion could influence the patient outcome.

\footnotetext{
* Correspondence: halamahfouz 1000@yahoo.com

${ }^{1}$ Menoufia University, P.O box 34, 55-El Gish street, Tanta, Egypt
}

Full list of author information is available at the end of the article
Earlier studies $[2,3]$ described the LAD anatomical course, and divisions are varied in approximately $78 \%$ of healthy individuals. The LAD route may extend around the apex and terminates beyond the diaphragmatic aspect of the left ventricle (LV). Nonetheless, LAD fails to continue on the diaphragmatic surface in $22 \%$ of patients; alternatively, it terminates at or even before the cardiac apex [4]. Further research work described the LAD coronary artery to be wrapped around the cardiac apex in $47 \%$ of individuals with right coronary artery dominance and in $87 \%$ of those with left coronary dominance [3]. 
Myocardial blood flow through LAD constitutes the largest cardiac blood supply that explains the poor patient's prognosis when proximal LAD occlusion developed. Proximal LAD occlusion would jeopardize a large portion of the myocardium and render the patient at a greater risk of heart failure and cardiac death [5-8]. The association between poor patients outcomes in anterior ST-segment elevation myocardial infarction (STEMI) and LAD anatomic features was lately explored $[9,10]$. During long-term follow-up, several investigators clarified the impact of LAD wrapping around the cardiac apex and the occurrence of adverse cardiovascular events due to the larger area of infarct $[9,10]$.

However, there is a lack of records in the literature as regards the relationship of LAD anatomical features and LV mechanics in patients with normal coronary angiography.

Two-dimensional (2D) strain imaging has been introduced as a novel method with angle-independency, acceptable reproducibility, and segmental quantification of LV strain $\left(\varepsilon_{\text {sys }}\right)$, strain rate (SR), and LV twist [11-14]; by these measurements, LV function could be assessed.

The aim of the present study was to examine functional characteristics of cardiac mechanics using twodimensional strain imaging (2D-strain) in patients with and without LAD wrapping around left ventricular apex in angiographically normal coronary arteries.

\section{Methods}

\section{Study population}

This is cross-sectional study included all patients who were scheduled for elective coronary angiography for the first time at our catheterization laboratory (university hospital) and have normal coronary angiography through the period from January 2016 to December 2018.

\section{Inclusion criteria}

Patients aged 18 years or more who were scheduled for elective coronary angiography and have normal coronary angiography presented with chest pain or referred for evaluation of coronary arteries to rule out coronary artery disease $(\mathrm{CAD})$ were enrolled in this study.

\section{The exclusion criteria}

Patients with evidence of obstructive CAD, previous acute coronary syndrome, prior coronary intervention/ coronary artery bypass graft surgery, anomalies of coronary arteries, moderate-to-severe valvular regurgitation or any valvular stenosis, heart failure, reduced ejection fraction (EF), left bundle branch block ( LBBB), atrial fibrillation, or malignancy were all excluded from the study. Written informed consent was obtained from the participants. The study proposal was approved by the Ethics Committee and the Research Board of the University.
Patients were recruited and examined in Yacoub Research Unit, in our university.

\section{Coronary angiogram}

All patients were subjected to elective coronary angiography using standard procedures and multiple projections and angles. Coronary arteries were assessed according to LAD termination character. "LAD wrapping" was defined as an LAD artery that perfuses at least one fourth of the LV inferior wall in the right anterior oblique and caudal projection, and it does not terminate at the apex but extends through the diaphragmatic surface. While LAD non-wrapping was defined when LAD does not expand on the diaphragmatic surface and terminates at the apex 1-4]. Angiographic data was reviewed by at least two experienced observers.

A normal coronary angiogram was defined as one with the absence of any identifiable pathology (comprises signs of atherosclerosis, spontaneous coronary spasm, or thrombosis) [1]. Entirely, patients had mild coronary artery atherosclerotic lesions with $\leq 50 \%$ diameter stenosis, were diagnosed as having an obstructive coronary disease and excluded from the study.

From 1600 patients who undergone elective angiography, 71 meet our inclusion criteria. The patients were categorized into two groups according to LAD anatomic feature: those with a long LAD (wrapping around LV apex) constituted (LAD wrapping group, $n=52$ ) and those with the LAD terminating at or before the apex constituted (LAD non-wrapping group, $n=19$ ).

\section{Conventional echocardiography}

All included patients underwent an echocardiographic examination within 1 week of coronary angiographic study using an ultrasonographic system (Esaote Mylab Gold 30 ultrasound system (Esaote S.p.A, Florence, Italy) equipped with a multi-frequency $2.5-3.5 \mathrm{MHz}$ phasedarray transducer was employed. LV end-diastolic and systolic (EDD, ESD) diameters, septum (SPT) and posterior (PWT) wall thickness, LV mass (LVM), and LVM index (LVMI) were calculated in accordance with the recommendations of the American Society of Echocardiography [15]. LV EF\% was measured using a modified Simpson's rule. Left atrial (LA) diameter and volume (LAV) were calculated using the biplane area-length method and indexed for BSA [15]. Measurements of pulmonary artery pressure (PAP) were carried out using tricuspid regurgitation velocity-derived Bernoulli equation $(\mathrm{PG}=4 \mathrm{~V} 2)$.

Mitral inflow velocity patterns were recorded from apical $4 \mathrm{CH}$ view $(4-5 \mathrm{~mm})$ sample volume width was utilized to be placed at the mitral leaflet tips, in diastole and using 3 consecutive cardiac cycles. An optimized pulsed wave Doppler beam parallel to the direction of 
mitral inflow was taken. Peak early and late diastolic trans-mitral velocities (E\&A) and E-wave deceleration time were measured. All pulsed Doppler signals were recorded at a horizontal sweep of $100 \mathrm{~mm} / \mathrm{s}$ [15].

LV diastolic function assessment was performed using conventional transmitral flow velocity-derived indices. Additionally, the mitral annular motion was recorded from the apical $4 \mathrm{CH}$ view using a tissue Doppler imaging program (TDI) [16]. A 4-5-mm sample volume was placed sequentially at the lateral and then septal corners of the mitral annulus. Peak systolic (S') early diastolic $\left(E^{\prime}\right)$ and late diastolic annular velocities were measured. Annular velocities were recorded during endexpiratory phase, at sweep speed 50 to $100 \mathrm{~mm} / \mathrm{s}$ and averaged from 3 consecutive cardiac cycles at both annuli [17]. E/E' as a measure of LV end-diastolic pressure was calculated $[15,17]$.

\section{Analysis of LV deformation}

Both LV segmental and global mechanics were analyzed using vector velocity imaging (VVI). It is an angleindependent feature-tracking mode that encompasses speckle tracking with endocardial and epicardial contour tracking. It measures myocardial motion from B-mode clips by automatically tracing contours to define the inward and outward myocardial motion [18].

Strain and SR measurements were recorded from apical $2 \mathrm{CH}$ and $4 \mathrm{CH}$ views at the frame rate $(90 \pm 20 \mathrm{~F} / \mathrm{s})$ according to the heart rate. The images and clips were stored, in digital format for subsequent offline analysis. Complete breath-holding during expiration was ensured to avoid excessive translational motion. Tracking and consequent strain analysis were performed using Esaote$\mathrm{X}$-Strain software package according to the validated algorithm [18]. Peak longitudinal systolic $\varepsilon_{\text {sys }}$, systolic SR $\left(\mathrm{SR}_{\text {sys }}\right)$, early $\left(\mathrm{SR}_{\mathrm{e}}\right)$, and atrial $\left(\mathrm{SR}_{\mathrm{a}}\right)$ diastolic $\mathrm{SR}$ of the basal, mid, and apical segments at the mid myocardial layer of septal, lateral, anterior, and inferior walls were measured and averaged to calculate the global deformation. Circ esys was measured from short-axis views at the mid papillary levels, midway between the mitral valve and the apex; LV twist was measured from shortaxis views at the basal and apical levels; the basal shortaxis plane contained the mitral valve; and the apical plane was acquired distally to the papillary muscle, as circular as possible and proximal to the plane with luminal obliteration at end-systole. LV twist was calculated as the instantaneous difference between apical and basal rotation $[18,19]$.

LV mechanical dyssynchrony, from regional strain curves the time to peak strain (TTP), was estimated for each ventricular segment, as the time from the beginning of the QRS complex of the ECG to the peak $\varepsilon_{\text {sys }}$. The electromechanical delay was measured as the difference between the longest and shortest TTP of the twelve LV myocardial segments [20]. LV dyssynchrony was defined as the standard deviation of the averaged TTP from all myocardial segments (TTP-SD) [20].

\section{Statistical analysis}

Values were presented as means \pm SD or as numbers and proportions, as appropriate. The relations between qualitative variables were evaluated by chi-square test or Fisher's exact test, as indicated. Means were compared with Student's $t$ test. Pearson correlation coefficient analysis was used to test the relation of LV twist and all clinical and echocardiographic variables. Variables that were significantly associated with patient's groups (wrapping versus non-wrapping) on univariate analysis were introduced into the multivariate logistic regression model to detect independent predictors. The analysis was performed by statistical package software IBMSPSS for MAC, version 25. All tests were bilateral and a $P$ value of $<0.05$ was considered statistically significant.

\section{Results}

In total, 71 patients having angiographically normal coronary arteries were included in the study; those with a long LAD wraparound LV apex constituted 73\% (LAD wrapping group, $n=52$ ) and those with the LAD terminating at or before LV apex constituted 27\% (LAD non-wrapping group, $n=19$ ).

\section{Patients' characteristics}

Patient's clinical characteristics were depicted in Table 1. No significant difference between study groups in age between LAD wrapping group $52 \pm 7.18$ and LAD nonwrapping group $50.4 \pm 8.7, P=\mathrm{NS}$; the male to female ratio was 1.53 [31 (59.6\%) males and $21(40.4 \%)$ females], among LAD wrapping group, and 1.36 [11 (57.9\%) males and 8 (42.1\%) females]. Patients with LAD wrapping have a higher prevalence of hypertension (62\% versus $26 \%, P<.008)$, lower prevalence of diabetes mellitus (59.6\% versus $100 \%, P<.001)$, and less prevalence of cigarette smoking $(16 \%$ versus $46 \%, P<.000)$ compared with LAD non-wrapping. No differences between patients groups in BSA, heart rate, blood pressure, or family history of CAD.

\section{Conventional echocardiographic findings}

There was no significant difference between the two groups in LV dimensions, LA dimension, $\mathrm{EF} \%$, or $\mathrm{E} / \mathrm{E}^{\prime}$ as demonstrated in Table 1. Meanwhile, LVM, LVMI, and DT were significantly higher in LAD wrapping compared with LAD non-wrapping group; LVM: $235.7 \pm$ 55.8 versus $193.3 \pm 34, P<0.003$; LVMI: $115.5 \pm 28$ versus $92.9 \pm 29, P<0.004$; and DT: $173.2 \pm 38.3$ versus $131.9 \pm 29.8, P<0.0001$. 
Table 1 Clinical and echocardiographic findings in studied groups

\begin{tabular}{|c|c|c|c|c|c|c|c|}
\hline & $\begin{array}{l}\text { LAD wrapping } \\
(\boldsymbol{n}=52)\end{array}$ & $\begin{array}{l}\text { LAD non-wrapping } \\
(\boldsymbol{n}=19)\end{array}$ & $\boldsymbol{P}$ value & & $\begin{array}{l}\text { LAD wrapping } \\
(\boldsymbol{n}=52)\end{array}$ & $\begin{array}{l}\text { LAD non-wrapping } \\
(\boldsymbol{n}=19)\end{array}$ & $\boldsymbol{P}$ value \\
\hline Age (year) & $52.13 \pm 7.18$ & $50.42 \pm 8.67$ & 0.40 & Apical Lat & $-13.21 \pm 5.3$ & $-13.01 \pm 2.5$ & 0.874 \\
\hline $\operatorname{BSA}\left(\mathrm{m}^{2}\right)$ & $1.99 \pm 0.13$ & $1.93 \pm 0.13$ & 0.084 & Mean Lat & $-15.09 \pm 6.1$ & $-15.29 \pm 3.8$ & 0.892 \\
\hline Hieght (cm) & $172.2 \pm 14$ & $167.7 \pm 19$ & 0.300 & Basalinferior & $-15.28 \pm 4.9$ & $-15.79 \pm 6.2$ & 0.725 \\
\hline Weight (kg) & $89.2 \pm 15.2$ & $88.11 \pm 25.3$ & 0.997 & Mid inferior & $-13.76 \pm 5.8$ & $-14.49 \pm 5.1$ & 0.634 \\
\hline BMI $\left(\mathbf{k g} / \mathrm{m}^{2}\right)$ & $1.72 \pm 0.15$ & $1.67 \pm 0.19$ & 0.542 & Apical inf & $-13.75 \pm 4.2$ & $-13.44 \pm 3.9$ & 0.779 \\
\hline Hypertension & $32(61.5 \%)$ & $5(26.3 \%)$ & .008 & Mean inf & $-14.27 \pm 4.5$ & $-14.57 \pm 4.6$ & 0.803 \\
\hline Diabetes mellitus & $31(59.6 \%)$ & 19(100\%) & .001 & Basal ant & $-17.32 \pm 6.9$ & $-15.9 \pm 3.9$ & 0.420 \\
\hline Smoking & $4(16.7 \%)$ & $6(46.2 \%)$ & .000 & Mid ant & $-14.62 \pm 7.4$ & $-15.04 \pm 2.4$ & 0.785 \\
\hline FH of CAD & $13(25 \%)$ & $4(21.1 \%)$ & .730 & Apical ant & $-13.77 \pm 3.8$ & $-14.73 \pm 2.2$ & 0.295 \\
\hline SBP $(\mathrm{mmHg})$ & $132 \pm 11$ & $132.4 \pm 12.1$ & 0.961 & Mean ant & $-15.23 \pm 5.0$ & $-15.26 \pm 2.6$ & 0.985 \\
\hline DBP $(\mathrm{mmHg})$ & $78.75 \pm 7.85$ & $75.79 \pm 6.72$ & 0.149 & Global & $-14.80 \pm 3.6$ & $-14.9 \pm 2.7$ & 0.836 \\
\hline HR (b/min) & $78.63 \pm 10.97$ & $81.39 \pm 6.31$ & 0.318 & TTP (ms) & $348.41 \pm 53$ & $339.1 \pm 32$ & 0.482 \\
\hline LAD (mm) & $37.06 \pm 3.58$ & $35.31 \pm 3.36$ & 0.73 & TTP-SD (ms) & $40.68 \pm 33.12$ & $30.06 \pm 32.5$ & 0.204 \\
\hline $\mathrm{LA} V\left(\mathrm{ml} / \mathrm{m}^{2}\right)$ & $52.52 \pm 15.77$ & $45.94 \pm 9.72$ & 0.102 & TTP-d (ms) & $131.3 \pm 91$ & $98.0 \pm 64.7$ & 0.161 \\
\hline $\operatorname{ESD}(\mathrm{mm}))$ & $29.96 \pm 3.75$ & $28.61 \pm 3.47$ & 0.184 & \multicolumn{4}{|c|}{ Longitudinal $\mathrm{SR}_{\text {sys }}\left(\mathrm{s}^{-1}\right)$} \\
\hline EDD (mm) & $47.4 \pm 4.7$ & $45.0 \pm 5.3$ & 0.074 & Septum & $-1.91 \pm 7.34$ & $-0.84 \pm 0.42$ & 0.53 \\
\hline Septum (mm) & $10.29 \pm 2.0$ & $9.70 \pm 2.4$ & 0.344 & Lateral & $-0.92 \pm 0.32$ & $-0.91 \pm 0.28$ & 0.79 \\
\hline PW (mm) & $10.28 \pm 1.9$ & $9.60 \pm 2.4$ & 0.213 & Anterior & $-0.92 \pm 0.31$ & $-1.06 \pm 1.36$ & 0.50 \\
\hline FS\% & $36.7 \pm 4.9$ & $37.2 \pm 5.1$ & 0.704 & Inferior & $-0.83 \pm 0.34$ & $-0.77 \pm 0.19$ & 0.46 \\
\hline $\mathrm{EF} \%$ & $65.1 \pm 9.91$ & $67.2 \pm 5.66$ & 0.405 & Global & $-1.17 \pm 2.02$ & $-0.89 \pm 0.36$ & 0.56 \\
\hline LVM (gm) & $235.7 \pm 55.8$ & $193.1 \pm 34.3$ & 0.003 & \multicolumn{4}{|c|}{ Longitudinal $\mathrm{SR}_{\mathrm{e}}\left(\mathrm{s}^{-1}\right)$} \\
\hline LVMI $\left(g m / m^{2}\right)$ & $115.5 \pm 28$ & $92.9 \pm 29$ & 0.004 & Mean septum & $1.01 \pm 0.40$ & $0.92 \pm 0.21$ & 0.345 \\
\hline Mitral E (cm/s) & $41.9 \pm 21.5$ & $50.3 \pm 12.9$ & 0.117 & Mean lateral & $1.03 \pm 0.47$ & $1 \pm 0.27$ & 0.819 \\
\hline Mitral A (cm/s) & $53.1 \pm 28$ & $59.9 \pm 11$ & 0.325 & Mean anterior & $1.01 \pm 0.46$ & $0.92 \pm 0.29$ & 0.395 \\
\hline Mitral E/A & $78.9 \pm 16.9$ & $0.84 \pm 0.19$ & 0.417 & Mean inferior & $1.00 \pm 0.38$ & $0.90 \pm 0.26$ & 0.257 \\
\hline DT (msec) & $172.2 \pm 38.4$ & $131.9 \pm 29.8$ & 0.000 & Global & $0.72 \pm 0.61$ & $0.39 \pm 0.30$ & 0.026 \\
\hline $\mathrm{E} / \mathrm{Ea}$ & $4.68 \pm 2.69$ & $5.36 \pm 1.7$ & 0.302 & \multicolumn{4}{|c|}{ Longitudinal $\mathrm{SR}_{\mathrm{a}}\left(\mathrm{s}^{-1}\right)$} \\
\hline Longitudinal $\varepsilon_{\text {sys }} \%$ & & & & Apical septum & $0.54 \pm 0.25$ & $0.42 \pm 0.14$ & 0.052 \\
\hline Basal septum & $-15.10 \pm 3.1$ & $-15.7 \pm 3.7$ & 0.561 & Septum & $0.85 \pm 1.72$ & $0.50 \pm 0.42$ & 0.374 \\
\hline Mid septum & $-14.67 \pm 5.4$ & $-15.1 \pm 3.1$ & 0.717 & Apical Lateral & $0.55 \pm 0.23$ & $0.39 \pm 0.11$ & 0.005 \\
\hline Apical septum & $-13.59 \pm 3.6$ & $-13.2 \pm 3.1$ & 0.679 & Lateral & $0.57 \pm 0.21$ & $0.45 \pm 0.16$ & 0.026 \\
\hline Mean septum & $-14.60 \pm 3.8$ & $-14.9 \pm 2.4$ & 0.798 & Apicalanterior & $0.53 \pm 0.24$ & $0.36 \pm 13$ & 0.004 \\
\hline basal lateral & $-16.17 \pm 8.6$ & $-17.0 \pm 5.9$ & 0.697 & Anterior & $0.61 \pm 0.26$ & $0.16 \pm 1.07$ & 0.006 \\
\hline Mid lateral & $-15.11 \pm 5.9$ & $-14.9 \pm 3.8$ & 0.880 & Global & $0.72 \pm 0.61$ & $0.39 \pm 0.30$ & 0.026 \\
\hline
\end{tabular}

$B S A$ body surface area, $B M I$ body mass index systolic blood pressure, $D B P$ diastolic blood pressure, $L A D$ left atrium dimension, $L A V$ left atrium volume, ESD left ventricular end-systolic diameter, EDD left ventricular end-diastolic diameter, $E F$ ejection fraction, $P W$ posterior wall, $L V M I$ left ventricular mass index, E/A ratio of early to late diastolic mitral inflow velocity, $D T$ deceleration time, E/Ea ratio of early diastolic mitral inflow velocity to early diastolic mitral annular velocity, $\varepsilon_{s y s}$ peak systolic strain, $S R_{\text {sys }}$ peak systolic strain rate, $S R_{e}$ early diastolic strain rate, $S R_{a}$ late diastolic strain rate

\section{Longitudinal strain}

There was no significant difference between LAD wrapping and LAD non-wrapping groups in segmental or global longitudinal strain $\left(\varepsilon_{\text {sys }}\right)$ or systolic strain rate $\left(\mathrm{SR}_{\mathrm{sys}}\right)$ as demonstrated in Table 1. Meanwhile, no significant difference between the two groups in electromechanical delay or mechanical dyssynchrony (TTP-d, TTP-SD) (Fig. 1).

In the LAD wrapping group, $\mathrm{SR}_{\mathrm{a}}$ of the apical septum $(P<.05)$, apical lateral $(P<.005)$, apical anterior $(P<$ $.004)$, lateral, and inferior was significantly greater than non-wrapping group $(P<.001)$. Consequently, the global 


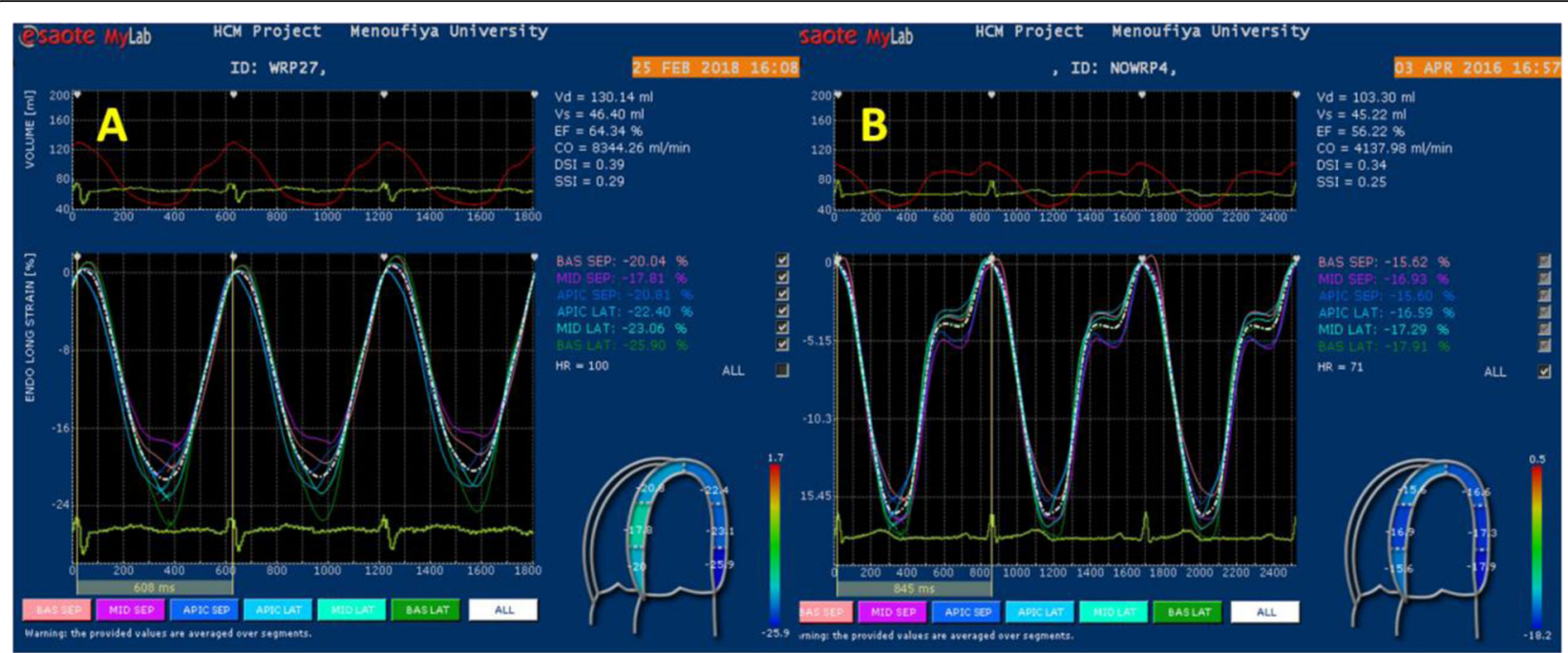

Fig. 1 Longitudinal strain (LS) curves derived from LV segments in apical $4 C H$ view. a $L A D$ wrapping patient with mean $L S=21 \%$ and $\mathbf{b} L A D$ non-wrapping with mean $\mathrm{LS}=17 \%$

atrial diastolic strain rate $\left(\mathrm{SR}_{\mathrm{a}}\right)$ was significantly higher in the LAD wrapping group compared with LAD nonwrapping group $P<0.02$ (Table 1).

\section{Circumferential strain}

Circumferential $\varepsilon_{\text {sys }} \%$ and SR were assessed at LV basal and apical planes in short-axis views (Tables 2 and 3). At basal level, LAD wrapping group showed higher circumferential $\mathrm{SR}_{\mathrm{a}}$ during late diastole at segmental level; lateral $(.54 \pm .23$ vs. $38 \pm .13, P<.005)$, posterior $(.50$ \pm .21 vs. $39 \pm .18, P<.03)$, inferior $(.53 \pm .19$ vs $.36 \pm .09$, $P<.0001)$, septum $(.49 \pm .15$ vs $.38 \pm .082, P<.005)$, and global levels $.74 \pm 1.14$ vs $.38 \pm .08, P<.01$. However, there was no significant difference in circumferential strain or SR during systole or early diastole (Figs. 2, 3 and 4).
The main finding of circumferential strain analysis at $L V$ apex was the higher values of circumferential $\varepsilon_{\text {sys }} \%$, $\mathrm{SR}_{\text {sys }}, \mathrm{SR}_{\mathrm{e}}$, and $\mathrm{SR}_{\mathrm{a}}$ in LAD wrapping group compared with LAD non-wrapping group; $\varepsilon_{\text {sys }} \%$ : $-15.92 \pm 4.46$ versus $-13.78 \pm 0.34, P<.02$; $\mathrm{SR}_{\text {sys }}$ : $-1.25 \pm 1.85$ versus $-0.84 \pm 0.13, P<.0001 ; \mathrm{SR}_{\mathrm{e}}: 1.09 \pm 0.49$ versus $0.88 \pm$ $0.18, P<.0001$; and $\mathrm{SR}_{\mathrm{a}}: .58 \pm .52$ versus $.39 \pm .062, P<$ .0001 .

In the LAD wrapping group, there was a significant increase in apical septal $(P<.01)$, apical anterior rotation with $P<.003$, and the averaged degree of LV apical rotation $(P<.003)$ compared with LAD non-wrapping group. These higher values of apical rotation resulted in augmented LV twist and LV torsion in LAD wrapping group compared with non-wrapping group $(P<.001$, $<.001$ ), respectively (Table 4, Figs. 5 and 6).

Table 2 Circumferential strain and strain rate at LV basal and apical segments

\begin{tabular}{|c|c|c|c|c|c|c|c|}
\hline LV basal & LAD wrapping & LAD non-wrapping & $\boldsymbol{P}$ value & LV apex & LAD wrapping & LAD non-wrapping & $\boldsymbol{P}$ value \\
\hline \multicolumn{8}{|l|}{ Circ $\varepsilon_{\text {sys }} \%$} \\
\hline Mean basal & $-13.87 \pm 4.0$ & $-13.5 \pm 3.2$ & 0.721 & Mean apical & $-15.92 \pm 4.5$ & $-13.78 \pm 0.3$ & 0.02 \\
\hline \multicolumn{8}{|l|}{$\operatorname{Circ} \operatorname{SR}_{\text {sys }}\left(s^{-1}\right)$} \\
\hline Mean basal & $-1.14 \pm 1.83$ & $-0.87 \pm .19$ & 0.533 & Mean apical & $-1.25 \pm 1.85$ & $-0.84 \pm .13$ & 0.000 \\
\hline \multicolumn{8}{|l|}{$\operatorname{Circ} \mathrm{SR}_{\mathrm{e}}\left(\mathrm{s}^{-1}\right)$} \\
\hline Mean basal & $0.96 \pm .44$ & $0.90 \pm .25$ & 0.564 & Mean apical & $1.09 \pm 0.49$ & $0.88 \pm .18$ & 0.000 \\
\hline \multicolumn{8}{|l|}{$\operatorname{Circ} \operatorname{SR}_{\mathrm{a}}\left(\mathrm{s}^{-1}\right)$} \\
\hline Basal septum & $0.49 \pm .15$ & $0.38 \pm .082$ & 0.005 & Apical anterior & $0.56 \pm .27$ & $0.44 \pm .14$ & 0.053 \\
\hline Basal anterior & $0.52 \pm .25$ & $0.41 \pm .19$ & 0.067 & Apico septal & $0.58 \pm .30$ & $0.37 \pm .10$ & 0.005 \\
\hline Basal lateral & $0.54 \pm .23$ & $0.38 \pm .13$ & 0.005 & Apical lateral & $0.5635 \pm .29$ & $0.39 \pm .10$ & 0.017 \\
\hline Basal inferior & $0.53 \pm .19$ & $0.36 \pm .09$ & 0.000 & Apical infer & $0.64 \pm .43$ & $0.36 \pm .073$ & 0.007 \\
\hline Mean basal & $0.74 \pm 1.14$ & $0.38 \pm .08$ & 0.0121 & Mean apical & $0.58 \pm .52$ & $0.39 \pm .062$ & 0.000 \\
\hline
\end{tabular}

$\varepsilon_{\text {sys }}$ peak systolic strain, $S R_{\text {sys }}$ peak systolic strain rat, $S R_{e}$ early diastolic strain rate, $S R_{a}$ late diastolic strain rate 
Table 3 LV rotation in studied groups

\begin{tabular}{llll}
\hline & LAD wrapping $(\boldsymbol{n}=52)$ & LAD non-wrapping $(\boldsymbol{n}=19)$ & $\boldsymbol{P}$ value \\
\hline ROT basal anteroseptal & $-2.65 \pm 2.25$ & $-2.22 \pm 1.64$ & .453 \\
ROT basal anterior & $-3.42 \pm 2.34$ & $-2.91 \pm 1.98$ & .404 \\
ROT basal lateral & $-4.22 \pm 1.96$ & $-3.39 \pm 2.90$ & .174 \\
ROT basal inferior & $-3.81 \pm 1.81$ & $-3.92 \pm 2.66$ & $-4.44 \pm 2.01$ \\
ROT basal posterior & $-3.88 \pm 2.45$ & $-3.64 \pm 1.78$ & .843 \\
ROT basal septum & $-3.82 \pm 2.58$ & $-3.41 \pm 2.12$ & .374 \\
Mean basal ROT & $-4.80 \pm 2.91$ & $2.69 \pm 1.56$ & .781 \\
ROT apical septum & $3.58 \pm 1.31$ & $2.57 \pm 1.10$ & .408 \\
ROT apical anterior & $3.62 \pm 1.32$ & $2.54 \pm 1.50$ & .019 \\
ROT apical lateral & $3.51 \pm 1.78$ & $3.70 \pm 1.89$ & .003 \\
ROT apical inferior & $4.52 \pm 1.79$ & $3.03 \pm 1.37$ & .087 \\
Mean apical ROT & $4.39 \pm 1.28$ & $73.87 \pm 15.57$ & .218 \\
Long axis & $70.37 \pm 13.83$ & $6.44 \pm 2.09$ & .003 \\
Twist & $8.91 \pm 3.19$ & $0.08 \pm 0.71$ & .518 \\
Torsion & $0.12 \pm 0.66$ & & .001 \\
\hline
\end{tabular}

\section{Relation of torsion and twist to clinical and echocardiographic variables}

Pearson correlation coefficient analysis was used to test the relation of LV twist and all clinical and echocardiographic variables. No significant correlation was verified between torsion and twist and any of clinical and conventional echocardiographic parameters.

On univariate analysis of the LV twist and LV longitudinal deformation (Fig. 7), LV twist showed a strong direct correlation to LV global $\boldsymbol{\varepsilon}_{\text {sys }}(r=.477, P<.000)$, modest inverse correlation to LV dyssynchrony as estimated by TTP-SD $(r=-.25, P<.03$, electromechanical delay as measured by TTP-d $(r=-.23, P<.03)$, and direct correlation to $\mathrm{LV}$ systolic $\mathrm{SR}\left(\mathrm{SR}_{\mathrm{sys}}\right)(r=.23, P<$ .05). Furthermore, the LV twist showed a strong direct correlation to diastolic function during early diastole as estimated by $\mathrm{SR}_{\mathrm{e}}(r=.55, P<.000)$. Considering circumferential strain, LV twist showed a strong direct correlation to both basal $(r=.56, P<.000)$ and apical rotation degree $(r=.62, P<.000)$ (Fig. 8). No significant correlation was detected between LV torsion and any of the deformation variables.

Variables that were statistically significant in univariate analysis were introduced in a forward logistic regression

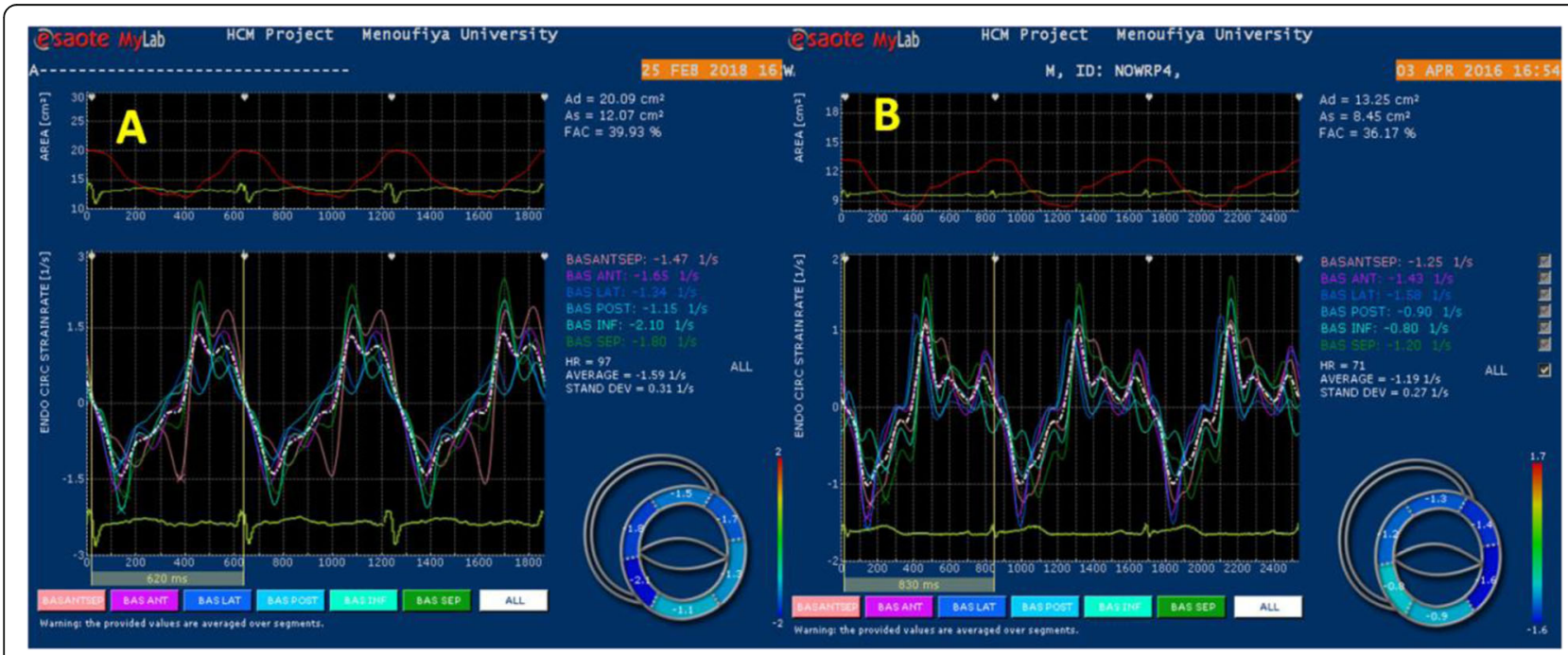

Fig. 2 LV circumferential strain rate curves derived from short axis view at mitral valve annulus. a LAD wrapping patient with mean basal Circ $\mathbf{S R}_{\mathbf{a}}$ $\mathbf{1 . 2 3}\left(\mathbf{s}^{-1}\right)$ and $\mathbf{b} L A D$ non-wrapping with mean basal Circ $\mathbf{S R}_{\mathbf{a}} \mathbf{0 . 4 3}\left(\mathrm{s}^{-}{ }^{-1}\right)$ 


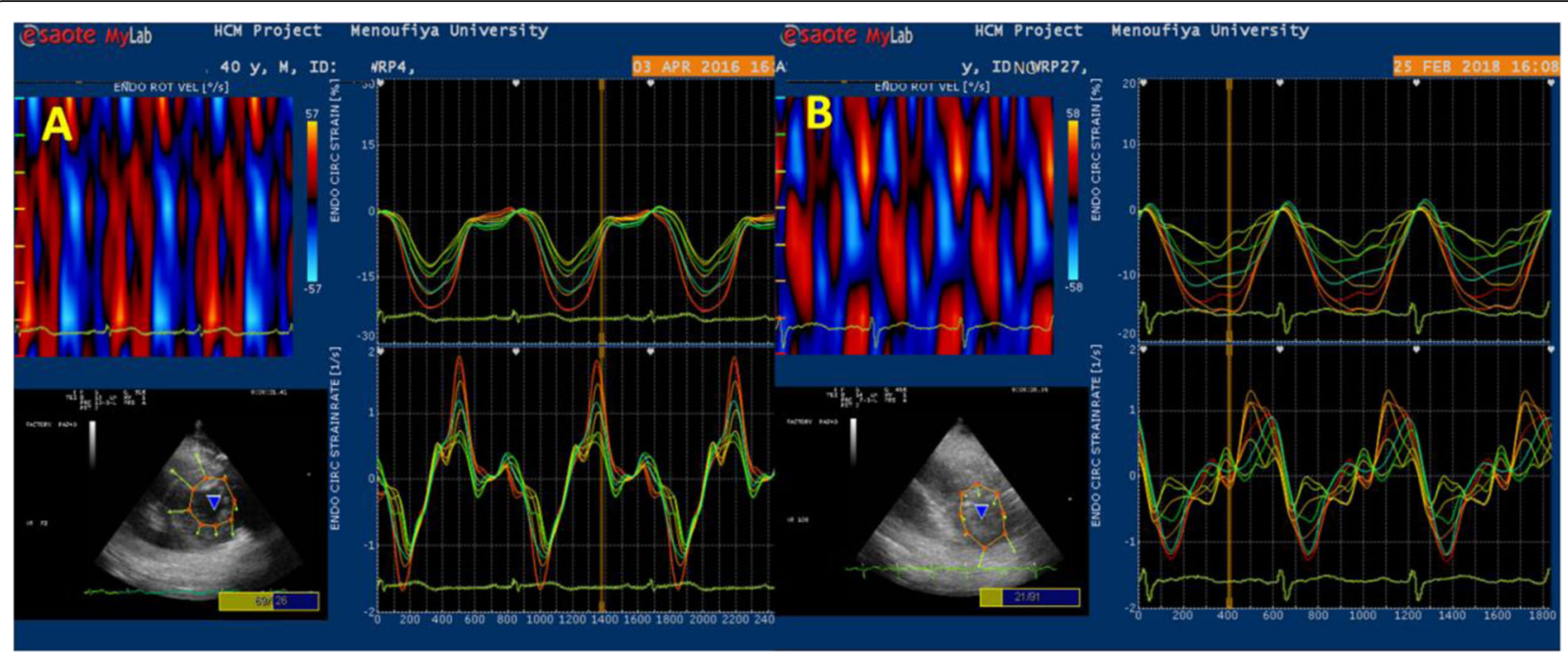

Fig. 3 Circumferential strain and strain rate curves using WI at short-axis view of LV apex. Upper left: curved M-mode of apical circ SR. Upper right: circ strain and lower right: circ SR curves. a LAD wrapping (mean value of circ $\varepsilon_{\text {sys }}=22 \%$ ). $\mathbf{b} L A D$ non-wrapping (mean value circ $\varepsilon_{5 y s}$ $=13 \%)$

model to estimate the independent predictors of LAD wrapping; these include hypertension diabetes mellitus, smoking, LVMI, DT, longitudinal $\mathrm{SR}_{\mathrm{a}}$, circumferential $\varepsilon_{\text {sys }}$ circ $S R_{\text {sys }}$, circ $S R_{e}$, circ $S R_{a}$, apical rotation, and $L V$ twist to discriminate LAD wrapping from LAD nonwrapping. From all these variables, LVMI [odds ratio .922, CI .860-.990, $P<.02$ ], DT [odds ratio .932, CI .877-991, $P<.02$ ], global longitudinal $\mathrm{SR}_{\mathrm{a}}$ [odds ratio $.000 \mathrm{CI} .000-.271, P<.03]$, and LV apical rotation [.931, CI $.876-.960$ and $P<.02$ ] were independent predictors for LAD wrapping around LV apex in angiographically normal coronary arteries.

\section{Discussion}

In the current study, we demonstrated that in patients with angiographically normal coronary arteries, long LAD that wrapped around LV apex ensured better longitudinal relaxation during late diastole, higher circumferential deformation at the LV apex, and augmented apical rotational mechanics. This deformational changes were resulting in higher LV twist despite no difference in LV ejection fraction measured by conventional methods. Longitudinal deformation in late diastole and LV apical rotation are independent predictors of LAD wrapping around LV apex in the presence of normal coronary circulation.

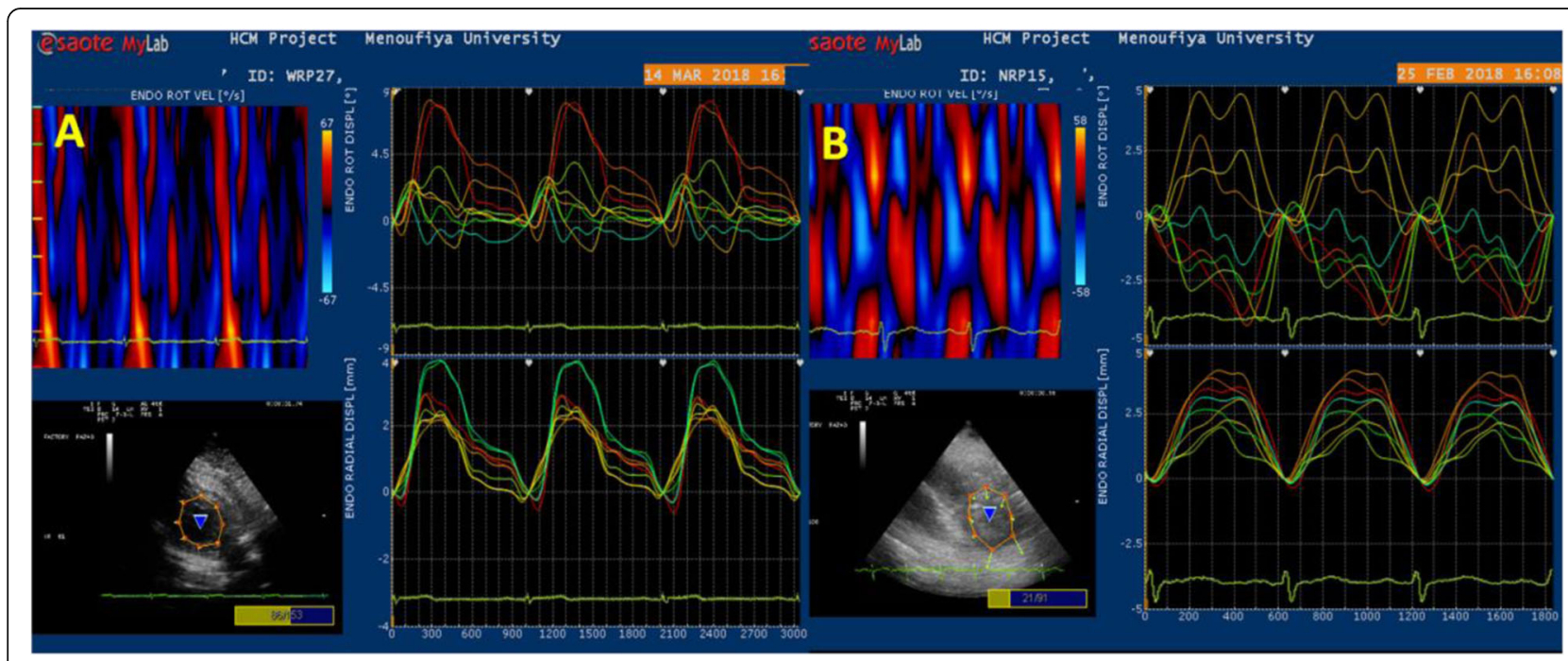

Fig. 4 Apical rotational mechanics: upper left: curved M-mode of rotation velocity $(\% / s)$. Upper right: rotation degree of $L V$ apex in a $L A D$ wrapping (mean value $=5.5^{\circ}$ ). $\mathbf{b} L A D$ non-wrapping (mean value $=3.5^{\circ}$ ) 
Table 4 Relationship of torsion and twist to clinical and echocardiographic variables

\begin{tabular}{|c|c|c|c|c|c|c|c|}
\hline & & Torsion & Twist & & & Torsion & Twist \\
\hline HR (b/min) & $\begin{array}{l}R \\
P\end{array}$ & $\begin{array}{l}-.014 \\
.905\end{array}$ & $\begin{array}{l}.084 \\
.487\end{array}$ & mitral E/A & $\begin{array}{l}R \\
P\end{array}$ & $\begin{array}{l}-.015 \\
.902\end{array}$ & $\begin{array}{l}.123 \\
.310\end{array}$ \\
\hline SBP $(\mathrm{mmHg})$ & $\begin{array}{l}R \\
P\end{array}$ & $\begin{array}{l}-.107 \\
.374\end{array}$ & $\begin{array}{l}-.168 \\
.162\end{array}$ & DT (ms) & $\begin{array}{l}R \\
P\end{array}$ & $\begin{array}{l}-.174 \\
.152\end{array}$ & $\begin{array}{l}-.011 \\
.927\end{array}$ \\
\hline $\mathrm{DBP}(\mathrm{mmHg})$ & $\begin{array}{l}R \\
P\end{array}$ & $\begin{array}{l}-.183 \\
.126\end{array}$ & $\begin{array}{l}-.119 \\
.323\end{array}$ & $E / E_{a}$ & $\begin{array}{l}R \\
P\end{array}$ & $\begin{array}{l}-.083 \\
.493\end{array}$ & $\begin{array}{l}-.147 \\
.225\end{array}$ \\
\hline LAD (mm) & $\begin{array}{l}R \\
P\end{array}$ & $\begin{array}{l}-.072 \\
.556\end{array}$ & $\begin{array}{l}-.001 \\
.993\end{array}$ & $E_{a}(\mathrm{~cm} / \mathrm{s})$ & $\begin{array}{l}R \\
P\end{array}$ & $\begin{array}{l}-.089 \\
.465\end{array}$ & $\begin{array}{l}.080 \\
.510\end{array}$ \\
\hline $\operatorname{LAV}\left(\mathrm{ml} / \mathrm{m}^{2}\right)$ & $\begin{array}{l}R \\
P\end{array}$ & $\begin{array}{l}-.101 \\
.405\end{array}$ & $\begin{array}{l}.048 \\
.690\end{array}$ & $A_{a}(\mathrm{~cm} / \mathrm{s})$ & $\begin{array}{l}R \\
P\end{array}$ & $\begin{array}{l}-.067 \\
.583\end{array}$ & $\begin{array}{l}.180 \\
.137\end{array}$ \\
\hline ESD (mm) & $\begin{array}{l}R \\
P\end{array}$ & $\begin{array}{l}-.055 \\
.652\end{array}$ & $\begin{array}{l}-.033 \\
.787\end{array}$ & $\mathrm{~S}_{\mathrm{a}}(\mathrm{cm} / \mathrm{s})$ & $\begin{array}{l}R \\
P\end{array}$ & $\begin{array}{l}.105 \\
.385\end{array}$ & $\begin{array}{l}.132 \\
.275\end{array}$ \\
\hline EDD (mm) & $\begin{array}{l}R \\
P\end{array}$ & $\begin{array}{l}-.090 \\
.460\end{array}$ & $\begin{array}{l}-.048 \\
.694\end{array}$ & $\varepsilon_{\text {sys }} \%$ Global & $\begin{array}{l}R \\
P\end{array}$ & $\begin{array}{l}.070 \\
.563\end{array}$ & $\begin{array}{l}.477 \\
.000\end{array}$ \\
\hline Septum (mm) & $\begin{array}{l}R \\
P\end{array}$ & $\begin{array}{l}.033 \\
.789\end{array}$ & $\begin{array}{l}-.042 \\
.727\end{array}$ & Mean TTP (ms) & $\begin{array}{l}R \\
P\end{array}$ & $\begin{array}{l}-.107 \\
.374\end{array}$ & $\begin{array}{l}-.188 \\
.116\end{array}$ \\
\hline PW (mm) & $\begin{array}{l}R \\
P\end{array}$ & $\begin{array}{l}-.002 \\
.990\end{array}$ & $\begin{array}{l}.001 \\
.993\end{array}$ & TTP-SD (ms) & $\begin{array}{l}R \\
P\end{array}$ & $\begin{array}{l}.163 \\
.175\end{array}$ & $\begin{array}{l}-.249 \\
.032\end{array}$ \\
\hline FS\% & $\begin{array}{l}R \\
P\end{array}$ & $\begin{array}{l}-.064 \\
.600\end{array}$ & $\begin{array}{l}.073 \\
.550\end{array}$ & TTP-d (ms) & $\begin{array}{l}R \\
P\end{array}$ & $\begin{array}{l}.170 \\
.157\end{array}$ & $\begin{array}{l}-.231 \\
.053\end{array}$ \\
\hline $\mathrm{EF} \%$ & $\begin{array}{l}R \\
P\end{array}$ & $\begin{array}{l}-.063 \\
.605\end{array}$ & $\begin{array}{l}-.065 \\
.591\end{array}$ & Global SR $\mathrm{R}_{\text {sys }}(\%)$ & $\begin{array}{l}R \\
P\end{array}$ & $\begin{array}{l}.007 \\
.952\end{array}$ & $\begin{array}{l}.231 \\
.053\end{array}$ \\
\hline LVM (gm) & $\begin{array}{l}R \\
P\end{array}$ & $\begin{array}{l}-.132 \\
.278\end{array}$ & $\begin{array}{l}.048 \\
.694\end{array}$ & Global $\mathrm{SR}_{\mathrm{e}}\left(\mathrm{S}^{-1}\right)$ & $\begin{array}{l}R \\
P\end{array}$ & $\begin{array}{l}-.058 \\
.628\end{array}$ & $\begin{array}{l}.553 \\
.000\end{array}$ \\
\hline LVMI $\left(\mathrm{gm} / \mathrm{m}^{2}\right)$ & $\begin{array}{l}R \\
P\end{array}$ & $\begin{array}{l}-.109 \\
.369\end{array}$ & $\begin{array}{l}.111 \\
.362\end{array}$ & Global $\mathrm{SR}_{\mathrm{a}}\left(\mathrm{S}^{-1}\right)$ & $\begin{array}{l}R \\
P\end{array}$ & $\begin{array}{l}.045 \\
.710\end{array}$ & $\begin{array}{l}.078 \\
.520\end{array}$ \\
\hline Mitral E (cm/s) & $\begin{array}{l}R \\
P\end{array}$ & $\begin{array}{l}-.047 \\
.699\end{array}$ & $\begin{array}{l}-.016 \\
.897\end{array}$ & Basal Circ $\varepsilon_{\text {sys }} \%$ & $\begin{array}{l}R \\
P\end{array}$ & $\begin{array}{l}.074 \\
.541\end{array}$ & $\begin{array}{l}.546 \\
.000\end{array}$ \\
\hline Mitral A $(\mathrm{cm} / \mathrm{s})$ & $\begin{array}{l}R \\
P\end{array}$ & $\begin{array}{l}.016 \\
.896\end{array}$ & $\begin{array}{l}-.090 \\
.458\end{array}$ & Apical Circ $S_{\text {sys }}$ & $\begin{array}{l}R \\
P\end{array}$ & $\begin{array}{l}-.027 \\
.820\end{array}$ & $\begin{array}{l}.616 \\
.000\end{array}$ \\
\hline
\end{tabular}

$S B P$ systolic blood pressure, $D B P$ diastolic blood pressure, $L A D$ left atrium dimension, $L A V$ left atrium volume, $E S D$ left ventricular end-systolic diameter, $E D D$ left ventricular end-diastolic diameter, EF ejection fraction, $P W$ posterior wall, $L V M I$ left ventricular mass index, E/A ratio of early to late diastolic mitral inflow velocity, $D T$ deceleration time, E/Ea ratio of early to early tissue doppler diastolic mitral flow, $E_{a}$ early diastolic annular velocity, $A_{a}$ late diastolic annular velocity, $S_{a}$ peak systolic annular velocity, $\varepsilon_{s y s}$ peak systolic strain, $\Pi T P$ time to peak strain, $S D$ standard deviation, $S R_{s y s}$ peak systolic strain rat, $S R_{e}$ early diastolic strain rate, $S R_{a}$ diastolic strain rate

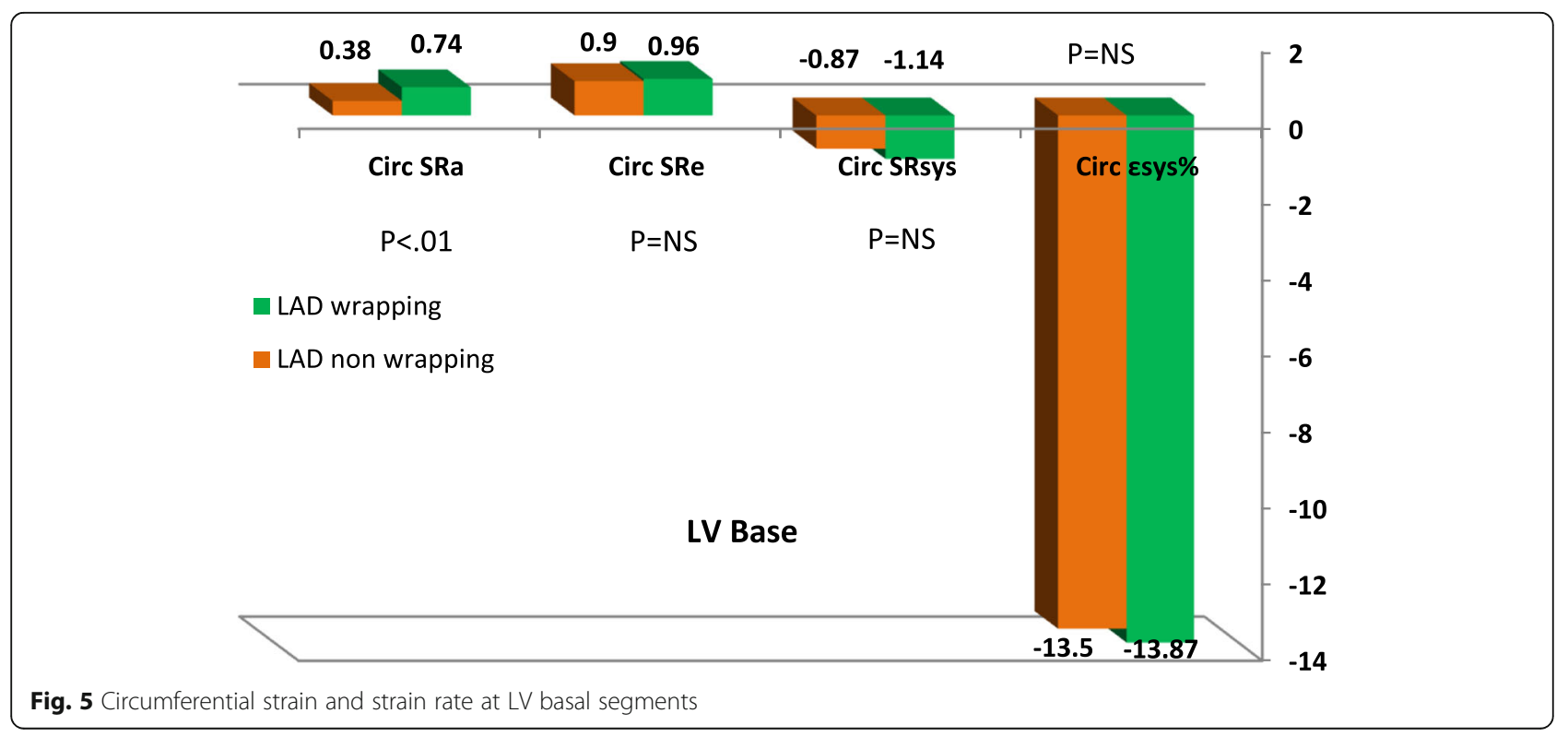




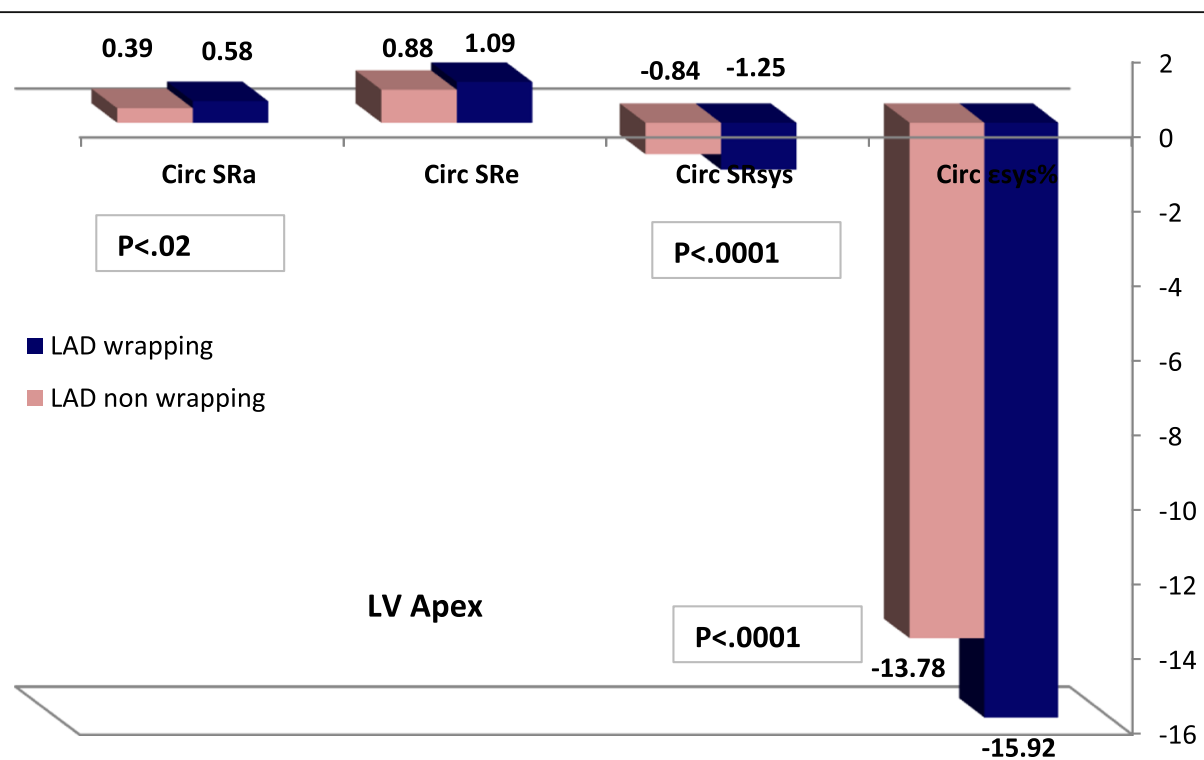

Fig. 6 Circumferential strain and strain rate at LV apex in study groups

The present study is the first study, up to our knowledge, that investigated the relationship between LAD coronary artery anatomical features and cardiac mechanics in patients with angiographically normal coronary arteries.

Evaluation of LV function using 2D Echo-Doppler primarily is unable to describe detailed information about cardiac mechanics, specifically when there are no coronary arterial or structural heart diseases. Strain imaging using VVI is a semi-automated technique that is more precise and accurate in characterizing LV function than the conventional methods.

Additionally, as distinct from previous studies, in our study, measurements of longitudinal and circumferential strain incorporated specifically the mid myocardial layer which reflecting the true regional function allowing an early detection of subtle myocardial abnormalities [18, 19].

Lately published researches verified the crucial value of global longitudinal strain (GLS) as an important functional parameter that provides imperative diagnostic and

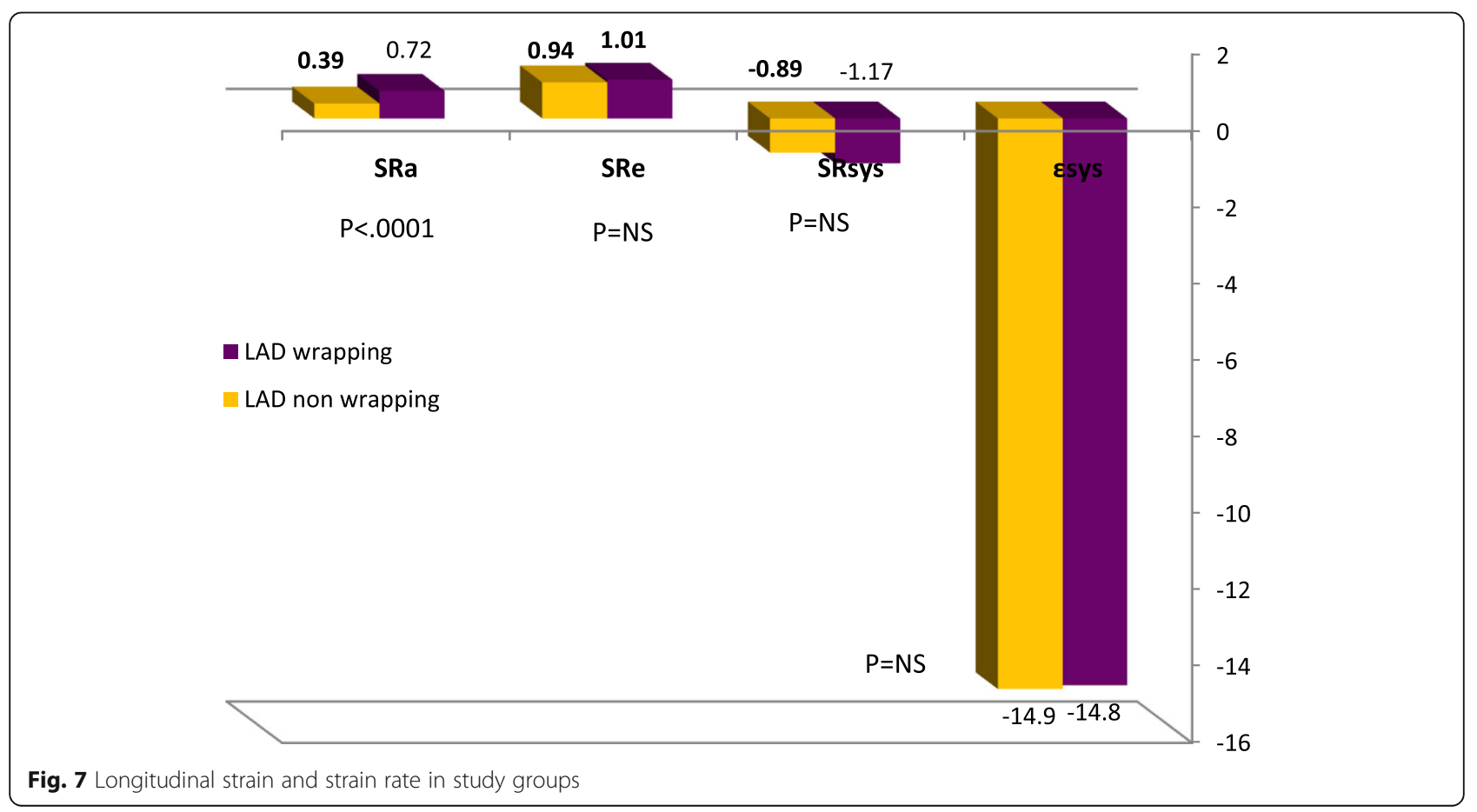




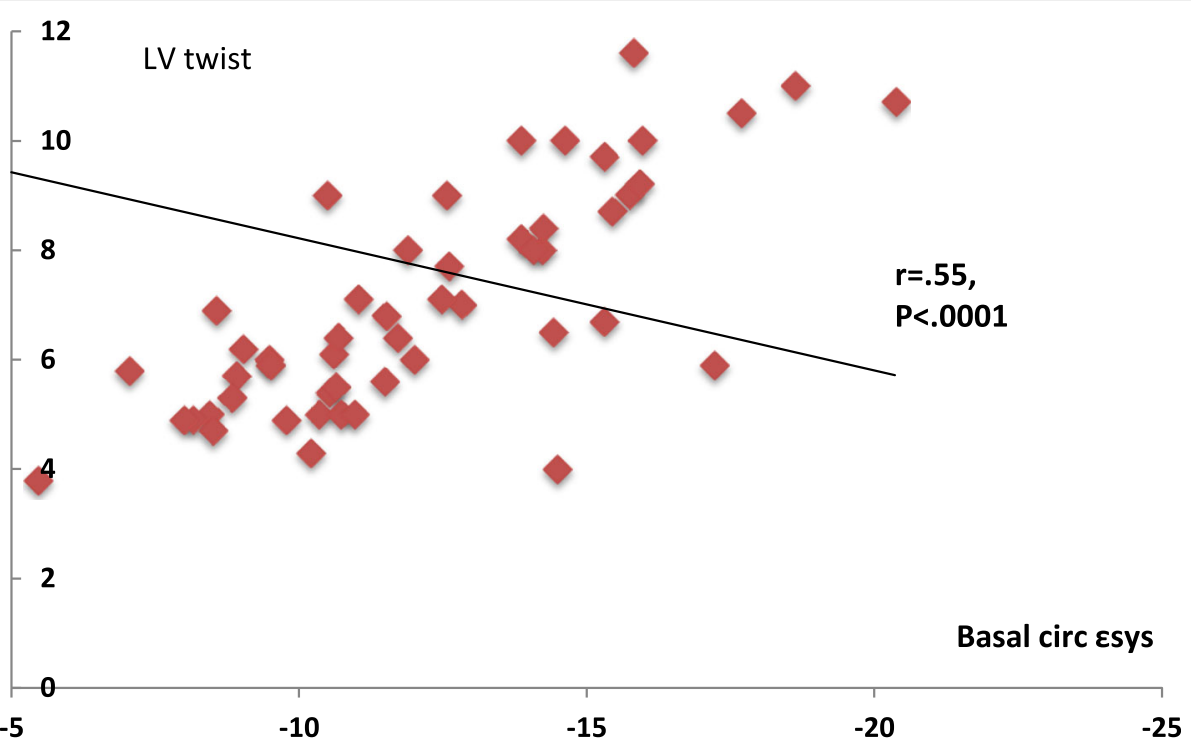

Fig. 8 Correlation of LV twist to circumferential strain at the basal segment

prognostic information in patients presented with acute coronary syndromes [21, 22], and it has also been demonstrated as an independent predictor of significant CAD in patients with chronic stable angina [23]. Liou et al. [24] stated that GLS might be an initial marker of CAD in symptomatic patients. Furthermore, Stankovic et al. [25] claimed that 2D strain imaging was an exceptional choice to detect LAD stenosis especially in an acute setting. In parallel with these studies, the present study verified increased circumferential strain during diastole can predict the length of LAD and its wrapping around the LV apex in absence of LAD stenosis.

Kobayashi et al. $[9,10]$ investigated the association between anatomic features of LAD and patient's outcome when presented with anterior ST-segment elevation myocardial infarction. They studied patients presented to $\mathrm{CCU}$ within $12 \mathrm{~h}$ after the development of suggestive symptoms of STEMI and subjected to primary percutaneous coronary intervention. Patients with LAD lesions confirmed to be the culprit lesions were divided into two groups, according to LAD anatomical features, as group 1: LAD wrapping $(n=871)$ and group 2 : LAD nonwrapping $(n=224)$. They demonstrated that heart failure, significant arrhythmias, and LV mural thrombi were more common in the wrap-around LAD group. Additionally, LV ejection fraction was worse in the wraparound LAD group $(54.5 \%$ versus $58.7 \%, P<0.006)$. Thereafter 3 years of follow-up, major adverse cardiac events (death, stroke/stent thrombosis) were higher (12.7\% versus $5.4 \%, P<0.002)$; death $(6.6 \%$ versus $3.2 \%$, $P<0.05)$, stroke $(1.9 \%$ versus $0.5 \%, P<0.12)$, stent thrombosis $(5.6 \%$ vs $2.3 \%, P=0.047)$, and severe heart failure $(4.5 \%$ versus $1.4 \%, P<0.03)$ were more prevalent in wrap-around LAD versus non-wrap-around LAD. Multivariate regression analysis revealed that LAD wrapping independently and considerably anticipated the occurrence of adverse cardiovascular events and severe heart failure in patients with an anterior STEMI.

In the current study, we perceived the explanation of different patient outcomes when LAD obstruction, with wrap-around apex, coexist as there is more loss of augmented function especially at LV apex; the LAD wrapping group had better mechanics and higher longitudinal relaxation. In addition, the main difference between wrapping and non-wrapping LAD groups is the higher circumferential function and augmented apical rotation that result in increased LV twist. So, not only a larger area of myocardium becomes vulnerable but a significant mechanical dysfunction is added when LAD obstruction supervenes, if LAD wraps around the apex.

Patients with proximal LAD occlusion are recognized by cardiac surgeons and cardiologists as a high-risk group with increased morbidity and mortality. However, not all proximal LAD lesions are of the same clinical relevance. Forty percent of LV myocardium is supplied by LAD, including the anterior wall and the anterior segment of the interventricular septum [26]. The LAD supplies all of this area, and much more when it wraps around the apex [3-5]. Therefore, according to our study, it is easy to understand now why proximal LAD disease can be a high-risk lesion. If LAD wrapping around the apex undergoes severe obstruction, not only a larger area of jeopardized myocardium is a consequence but also a loss of augmented LV function especially the apical rotational mechanics and the resulting LV twist. 
During systole, the LV undergoes a unique twist motion with a counterclockwise rotation at the apex and a clockwise rotation at the base. The resulting twist during peak systole is $7.7 \pm 3.5^{\circ}$ [27]. This is followed immediately by rapid untwist at the end of the systole. This unique LV twisting is considered to be an important playing role not only in systolic but also in the diastolic function. LV twist generates positive torsional deformation forces that develop in the subepicardium layer that can be added to the opposing negative torsional motion originating in the subendocardium [27]. Eventually, the torque in the subepicardium impacts subendocardial deformation and the whole wringing motion of the heart will be affected with the reduction of rotational mechanics.

In our study, LV apical rotation and overall LV twist are much lower in LAD non-wrapping, and the larger part of LV twist is generated from apical counterclockwise rotation, so the peak LV twist is predominantly reduced in apical myocardial infarction; this might explain the reduction of LV torsion and even the occurrence of an apical aneurysm in apical myocardial infarction [28, 29].

Yet, in the present study, longitudinal strain did not differ between the study groups, and the longitudinal strain caused by the contraction of myocardial tissue in the endocardial layer and has a high sensitivity to myocardial ischemia which does not exist in our patients with angiographically normal coronaries. Several studies demonstrated that global longitudinal strain is a more sensitive and robust parameter for evaluating myocardial function compared to circumferential and radial strain $[30,31]$, while in normal myocardium, the circumferential strain is better reflecting both systolic and diastolic function [30, 31].

In addition, our study demonstrated that the left ventricular diastolic function as measured by deceleration time, longitudinal and circumferential strain, and strain rate during diastole $\left(\mathrm{SR}_{\mathrm{a}}\right.$ and $\left.\mathrm{SR}_{\mathrm{e}}\right)$ was enhanced in a patient with long LAD that wraps around the apex. The underlying mechanism was reported in Wang et al.'s research [32]. Myocardial perfusion occurs mostly in diastole because systolic contraction transiently prevents coronary blood flow, especially to the subendocardium. So vascular turgor and large blood supply especially, to cardiac apex, will better perfuse the normal myocardium and predominantly augment the diastolic function. This diastolic function is initially affected if coronary artery stenosis develops.

Our study is the first to address the relationship between LAD length and LV function. We found that long LADs (i.e., those in which the vessel wraps around the apex) had higher values of LV function especially during diastole. From previous studies, LAD has a major impact on prognosis in patients undergoing primary PCI. This is probably as a result of a greater amount of the muscle supplied by this type of LAD, and therefore, a greater amount of myocardial necrosis when the vessel totally occludes $[9,10]$. Furthermore, the augmented LV function associated with LAD will be regained after revascularization. This claim is further strengthened in previous studies when no differences were found between the LAD wrapping and LAD non-wrapping groups with regard to the presence and extent of collaterals and the blush grade (reflecting myocardial perfusion) in patients after LAD revascularization, and LAD length was the main determinant after intervention [8, 33].

Notably, our study showed a strong positive correlation between the LV twist and longitudinal and circumferential strain and strain rate especially at LV apex, and this explains the augmented LV twist in the LAD wrapping group was associated with better circumferential strain values compared to the LAD non-wrapping group. We could expect that proximal LAD stenosis in such a group with LAD wrapping to further deteriorate LV function to a greater extent and recommend utilization of longitudinal and circumferential strain as an early marker of LAD stenosis that better reflect LV dysfunction than LV EF\%. This is in agreement with Stokke et al. [34] who reported a study showing strain imaging probably better reflects systolic function in patients with a preserved estimated LVEF.

There are numerous factors such as age, sex, diabetes mellitus, and hypertension that can affect cardiac mechanics in our study population, as examined by longitudinal and circumferential strain. Tadic et al. [35] demonstrated that patients with non-complicated diabetes and hypertension also had impaired LV longitudinal strain. In the present study, the prevalence of hypertension was higher in the LAD wrapping group while diabetes mellitus and smoking were more prevalent in LAD non-wrapping group which makes both groups at equal risk. Moreover, the ratio of male to female patients was similar in the study population, so the role of these risk factors on cardiac mechanics cannot be used to explain the difference in cardiac mechanics.

We included a real-life patient's population with a variable risk profile who was referred to the catheterization laboratory for coronary angiography. However, we thought that the impact of these risk factors on cardiac mechanics might be equivalent. In the LAD wrapping group, hypertension was prevalent in $60 \%$ while diabetes mellitus and smoking were prevalent in $100 \%$ and $46 \%$ in LAD nonwrapping which represents a balanced exposure to cardiovascular risk. Compensatory left ventricular hypertrophy was existent in only $35 \%$ of the LAD wrapping group which renders their LVMI $115 \mathrm{~g} / \mathrm{m}^{2}$ close to the normal values. Moreover, previous investigators reported that the longitudinal LV strain is decreased in patients with 
hypertension [13,36] whereas our study group showed no significant difference in longitudinal strain, between LAD wrapped and non-wrapped patients which preclude the influence of hypertensive left ventricular hypertrophy on cardiac mechanics that characterize LAD wrapping group.

\section{Limitations}

The present study has several limitations. First, it is a small sample-sized study with a limited number of patients; however, it can be explained by the strict exclusion criteria and single-center study. Second, image acquisition and analysis depend on many factors including image quality, operator experience especially with strain analysis software, and vendor variability. Third, concurrent risk factors like diabetes and hypertension might influence data results, despite invasive detection of LAD anatomical features in a healthy individual may be challenging. Finally, long-term follow-up could be performed to obtain clinical and prognostic figures especially when LAD obstruction occurs. Thus, large scale, multicenter, prospective, trials with enough power to examine the effect of all confounding factors are required to overcome these limitations.

\section{Conclusion}

LAD wrapping around LV apex is a characteristic anatomical feature of coronary circulation that provides better myocardial relaxation, augmented circumferential, and rotational mechanics contrast to non-wrapping feature, in patients with normal coronary angiography. This could explain the poor prognosis and worse outcome in such population when acute LAD occlusion supervenes.

\section{Abbreviation \\ 2D: Two-dimensional; CAD: Coronary artery disease; Circ: Circumferential; GLS: Global longitudinal strain; LAD: Left anterior descending artery; LV: Left ventricular; $\mathrm{SR}_{\mathrm{a}}$ : Atrial diastolic strain rate; $\mathrm{SR}_{\mathrm{e}}$ : Early diastolic strain rate; $\mathrm{SR}_{\text {sys: }}$ Systolic strain rate; STEMI: ST-segment elevation myocardial infarction; TTP-d: Electromechanical delay; TTP-SD: Standard deviation of time to peak; Wl: Vector velocity imaging; $\varepsilon_{\text {sys: }}$ : Peak systolic strain}

\section{Acknowledgements}

None

\section{Authors' contributions}

HB carried out the study design, statistical analysis, and writes the manuscript. Dr. WI participated in the clinical and echocardiographic study. Dr. TA participated in clinical examination and data collection, and Dr. GS participated in patient recruitments, clinical examination, and echocardiographic study. The authors read and approved the final manuscript

\section{Funding}

None

\section{Availability of data and materials}

The dataset supporting the results and conclusions of this article will be available from the corresponding author on request.

\section{Ethics approval and consent to participate}

This research involved human subjects and was performed in accordance with the Declaration of Helsinki and approved by the Menoufia University
Ethical Committee with reference number 3072016, Egypt. A written informed consent was obtained from all patients.

Consent for publication

Not applicable

\section{Competing interests}

The author Prof Hala Mahfouz Badran is the Editor-in-Chief of this journal and she declares that she has no competing interest and no one of the authors has competing interests regarding this manuscript. The authors declare that they have no competing interests.

\section{Author details}

${ }^{1}$ Menoufia University, P.O box 34, 55-El Gish street, Tanta, Egypt. ${ }^{2}$ National Heart Institute, Cairo, Egypt.

Received: 13 March 2020 Accepted: 27 April 2020

Published online: 03 June 2020

\section{References}

1. Yılmaztepe MA, Uçar FM (2018) Layer-specific strain analysis in patients with suspected stable angina pectoris and apparently normal left ventricular wall motion. Cardiovascular Ultrasound 16:25 https://www.ncbi.nlm.nih.gov/ pmc/articles/PMC6158830/

2. - -llia R. (1994) Proximal left anterior descending artery stenosis: dangerous or not?. Cathet Cardiovasc Diagn;33:101-102. https://www.ncbi.nlm.nih.gov/ pmc/articles/PMC6158830/

3. Ilia R, Rosenstein G, Weinstein JM et al (2001) Left anterior descending artery length in left and right coronary artery dominance. Coron Artery Dis 12:77-78

4. Perlmutt LM, Jay ME, Levin DC (1983) Variations in the blood supply of the left ventricular apex. Invest Radiol 18:138-142

5. Mahmarian JJ, Pratt CM, Boyce TM et al (1991) The variable extent of jeopardized myocardium in patients with single vessel coronary artery disease: quantification by thallium-201 single photon emission computed tomography. J Am Coll Cardiol 17:355-362

6. Kalbfleisch H, Hort W (1977) Quantitative study on the size of coronary artery supplying areas postmortem. Am Heart J 94:183-188

7. Klein LW, Weintraub WS, Agarwal JB et al (1986) Prognostic significance of severe narrowing of the proximal portion of the left anterior descending coronary artery. Am J Cardiol 58:42-46

8. Antoni ML, Hoogslag GE, Boden H et al (2012) Cardiovascular mortality and heart failure risk score for patients after ST-segment elevation acute myocardial infarction treated with primary percutaneous coronary intervention (Data from the Leiden MISSION! Infarct Registry). Am J Cardiol 109:187-194

9. Kobayashi N, Maehara A, Mintz GS et al (2015) Usefulness of the left anterior descending artery wrapping around the left ventricular apex to predict adverse clinical outcomes in patients with anterior wall ST-segment elevation myocardial infarction (an INFUSE-AMI Substudy). Am J Cardiol. 115(10):1389-1395

10. Kobayashi N, Maehara A, Brener SJ et al (2015) Usefulness of the left anterior descending coronary artery wrapping around the left ventricular apex to predict adverse clinical outcomes in patients with anteriorwall STsegment elevation myocardial infarction (from the harmonizing outcomes with revascularization and stents in acute myocardial infarction trial). Am J Cardiol. 116(11):1658-1665. https://doi.org/10.1016/j.amjcard.2015.09.004 Epub 2015 Sep 11

11. Notomi Y, Lysyansky P, Setser RM et al (2005) Measurement of ventricular torsion by two-dimensional ultrasound speckle tracking imaging. J Am Coll Cardiol 45:2034-2041

12. Helle-Valle T, Crosby J, Edvardsen T et al (2005) New noninvasive method for assessment of left ventricular rotation: speckle tracking echocardiography. Circulation 112:3149-3156

13. Huang J, Yan ZN, Rui YF et al (2016) Left ventricular systolic function changes in primary hypertension patients detected by the strain of different myocardium layers. j 95:e2440

14. Huang J, Yan ZN, Rui YF et al (2018) Left ventricular short-axis systolic function changes in patients with hypertrophic cardiomyopathy detected by two-dimensional speckle tracking imaging. BMC Cardiovasc Disord 18:13 
15. Mitchell C, Rahko PS, Blauwet LA et al (2019) Guidelines for performing a comprehensive transthoracic echocardiographic examination in adults: recommendations from the American Society of Echocardiography. Jam Soc Echocardiogr 32(1):1-64

16. -Murase M (2016) Assessing ventricular function in preterm infants using tissue Doppler imaging. Expert Rev Med Devices. ;13(4):325-338. doi: https:// doi.org/10.1586/17434440.2016.1153966. Epub Mar 7.

17. Nagueh SF, Appleton CP, Gillebert TC et al (2009) Recommendations for the evaluation of left ventricular diastolic function by echocardiography. Eur J Echocardiogr 10(2):165-193

18. Yingchoncharoen T, Agarwal S, Popovic ZB et al (2013) Normal ranges of left ventricular strain: a meta-analysis. J Am Soc Echocardiogr 26(2):185-191

19. Papadopoulou E, Kaladaridou A, Agrios J et al (2014) Factors influencing the twisting and untwisting properties of the left ventricle during normal pregnancy. Echocardiography 31:155-163

20. Badran HM, Elnoamany MF, Soltan G et al (2012) Relationship of mechanical dyssynchrony to QT interval prolongation in hypertrophic cardiomyopathy. Eur Heart J Cardiovasc Imaging. 13(5):423-432

21. Caspar T, Samet H, Ohana M et al (2017) Longitudinal 2D strain can help diagnose coronary artery disease in patients with suspected non-STelevation acute coronary syndrome but apparent normal global and segmental systolic function. Int J Cardiol. 236:91-94

22. Meimoun $\mathrm{P}, \mathrm{Abouth} \mathrm{S}, \mathrm{Clerc} J$ et al (2015) Usefulness of two-dimensional longitudinal strain pattern to predict left ventricular recovery and in-hospital complications after acute anterior myocardial infarction treated successfully by primary angioplasty. J Am Soc Echocardiogr 28(11):1366-1375

23. Biering-Sorensen T, Hoffmann S, Mogelvang R et al (2014) Myocardial strain analysis by 2-dimensional speckle tracking echocardiography improves diagnostics of coronary artery stenosis in stable angina pectoris. Circ Cardiovasc Imaging. 7(1):58-65

24. Liou K, Negishi K, Ho S et al (2016) Detection of obstructive coronary artery disease using peak systolic global longitudinal strain derived by twodimensional speckle-tracking: a systematic review and meta-analysis. J Am Soc Echocardiogr. 29(8):724-735 e724

25. Stankovic I, Putnikovic B, Cvjetan R et al (2015) Visual assessment vs. strain imaging for the detection of critical stenosis of the left anterior descending coronary artery in patients without a history of myocardial infarction. Eur Heart J Cardiovasc Imaging. 16(4):402-409

26. Edwards WD, Tajik AJ, Seward JB (1981) Standardized nomenclature and anatomic basis for regional tomographic analysis of the heart. Mayo Clin Proc 56:479-497

27. Tavakoli V, Sahba N (2013) Assessment of age-related changes in left ventricular twist by 3-dimensional speckle-tracking echocardiography. J Ultrasound Med 32(8):1435-1441

28. Takigiku K, Takeuchi M, Izumi C et al (2012) Normal range of left ventricular 2-dimensional strain: Japanese Ultrasound Speckle Tracking of the Left Ventricle (JUSTICE) study. Circ J. 76(11):2623-2632

29. Reisner SA, Lysyansky P, Agmon Y et al (2004) Global longitudinal strain: a novel index of left ventricular systolic function. J Am Soc Echocardiogr 17(6):630-633

30. Manovel A, Dawson D, Smith B et al (2010) Assessment of left ventricular function by different speckle-tracking software. Eur J Echocardiogr 11(5): 417-421

31. Koopman LP, Slorach C, Hui W et al (2010) Comparison between different speckle tracking and color tissue Doppler techniques to measure global and regional myocardial deformation in children. J Am Soc Echocardiogr 23(9):919-928

32. Wang D, Sun JP, Lee AP et al (2015) Evaluation of left ventricular function by three-dimensional speckle-tracking echocardiography in patients with myocardial bridging of the left anterior descending coronary artery. J Am Soc Echocardiogr. 28(6):674-682

33. Kandzari DE, Tcheng JE, Gersh BJ, CADILLAC Investigators et al (2006) Relationship between infarct artery location, epicardial flow, and myocardial perfusion after primary percutaneous revascularization in acute myocardial infarction. Am Heart J 151:1288-1295

34. Stokke TM, Hasselberg NE, Smedsrud MK et al (2017) Geometry as a confounder when assessing ventricular systolic function: comparison between ejection fraction and strain. J Am Coll Cardiol. 70(8):942-954

35. -Tadic M, Cuspidi C, Vukomanovic V, et al (2018) Layer specific deformation of the left ventricle in uncomplicated patients with type 2 diabetes and arterial hypertension. Arch Cardiovasc Dis.;111(1):17-24.
36. Kim SA, Park SM, Kim MN et al (2016) Assessment of left ventricular function by layer-specific strain and its relationship to structural remodelling in patients with hypertension. Can JCardiol 32:211-216

\section{Publisher's Note}

Springer Nature remains neutral with regard to jurisdictional claims in published maps and institutional affiliations.

\section{Submit your manuscript to a SpringerOpen ${ }^{\circ}$ journal and benefit from:}

- Convenient online submission

- Rigorous peer review

- Open access: articles freely available online

- High visibility within the field

- Retaining the copyright to your article

Submit your next manuscript at $\boldsymbol{\nabla}$ springeropen.com 